\title{
Manufacturing Digitalization and Its Effects on Production Planning and Control Practices
}

\author{
Siavash H. Khajavi ${ }^{1}$ and Jan Holmstrm ${ }^{1}$ \\ Department of Industrial Engineering and Management, Aalto University, Espoo, \\ Finland \\ siavash.khajavi, jan.holmstrom Qaalto.fi
}

\begin{abstract}
Advent of additive manufacturing (AM) as a final-parts production method has the capacity to impact the supply chains radically (The Economist, 2012). This effect extends from raw material procurement to production management and further towards distribution and the final customers. Digitalization of production as for the other industries such as automotive and aerospace reduces the operational complexity, while embedding the complexity in the digital components of the system. For instance, the production planning and control for an AMenabled manufacturing may be distinctly different compared to conventional production methods. Production routing, loading and scheduling can become simplified as steps of production are combined through AM utilization. Moreover, production dispatching, reporting, inspection and corrective actions require development of novel effective practices. In this paper we investigate the in-depth impact of digital production technologies (e.g. additive manufacturing) on the production management practices. Our methodology is based on conceptual modelling intertwined with case data.
\end{abstract}

Key words: Production planning and control, manufacturing digitalization, supply chain simplification

\section{Introduction}

The development of production systems introduced batching methods, standardization of components and stock keeping units to improve the efficiency of the production systems, however the emergence of computers and digitalization of tasks, changed the production methods and visibility of operations by improving the data gathering and sharing methods [7]. Now that the manufacturing machines becoming more and more software based and flexible, the question is do we still need the concepts previously set for the labor managed operations. We see a transition from a full human operated world towards a human-machine operated world within which machines are smarter and embed the know-how. In the new paradigm the order can be accomplished by an autonomous system to reach the outcome. 
What we looked into throughout this research is the possible impact of direct digital manufacturing technologies (including additive manufacturing) on the conventional production practices. If the conventional shop floor concepts such as batching, SKUs, and other production planning and control tactics can be necessary for the new paradigm of digital smart production. The method is to review the conventional practices point out the costly ones then realize the possibility to omit them through the new tagging and other methods, while using available case studies.

The remainder of this paper is organized as follows: Section 2 presents a literature review; Section 3 explains the research methodology; and Section 4 presents the findings and results of our analysis. This paper ends with conclusions summarizing the research outcomes, and suggestions for future investigation are provided.

\section{Literature Review}

In this section we briefly review the literature on the two main subjects of this paper which are direct digital manufacturing and production planning and control.

\subsection{Direct digital manufacturing}

Direct Digital Manufacturing (DDM) technologies are a set of novel manufacturing techniques that produce the final parts from a computer aided design (CAD) file. These technologies which started to appear during the 1980s also have been known as additive manufacturing and 3D printing production techniques. In most of these technologies the computer software slices the three dimensional model of the part to very thing two dimensional cross sections and send them one at a time to the printing machines. The machine then utilized various technologies to produce these parts a layer at a time to reach the final geometrical form factor $[1,10]$.

The application of these CAD based technologies in the beginning was focused on the production of design models and prototypes. However, as the material range expanded and production quality and precision increased the application shifted to functional prototypes and recently final parts. Still there are obstacles to overcome before this method of production can enter the mainstream use and realize a wide application. For instance the cost intensiveness of the production machines as well as raw material, low throughput of the processes, limited range of available material and labour intensiveness of the pre and post production steps are among the most notable issues that need to be addressed [2]. 
However, DDM has a number of unique characteristics which makes it a very useful manufacturing method for a number of industries even at the current cost levels. DDM enables the production of very small batch size (as small as the batch of one in medical applications) while also removes the design for manufacturing limitations to a high extent. This means the production of very complex parts with moving parts is possible in a single run, which translates to designers freedom to take advantage of design for performance instead. This is very relevant especially to the aerospace industry which searches for any opportunity to cut weight from the components and increase the fuel efficiency of airplanes. The other important features of this production method are the significant reduction in the amount of raw material waste through the production, the nonexistence of economics of scale (no tooling) and high flexibility for modifications on the fly $[1,9]$.

As the DDM attracts more attention and investment the new advancements will emerge which may turn it to a common production method for small and medium volume production batches in distributed settings which also has the potential to enable on-shoring of the manufacturing to the worlds developed countries and cut the costs of transportation of goods significantly [1]. Therefore we see the urge to study the impact of this technology on various aspect of production management to illustrate the potentials and refute the myths.

\subsection{Production planning and control}

Production planning and control (PPC) are a set of activities which have been designed to make the production and delivery of products more smooth and efficient both time and cost-wise. Planning sets the goals for the productions long, medium and short term goals with regard to product and processes, in order to satisfy the customers needs on time and uninterrupted. Production control is responsible to check the performance of the organization with regard to its production schedules and deliveries to alert any sign of derailment from the established plans. Moreover, the healthy functioning PPC can keep the inventories in check and customers satisfied through the optimum utilization of labor, material and machinery $[7,8]$.

Planning, routing, scheduling and loading are the main tasks in the production planning while, dispatching, follow up, inspection and corrective action are the production control practices. Planning is concerned with the processes which are required to be done to enable the production of the product, routing is the task of determining a path for the movement of the production lots in the factory while it is being made. Loading of the works on the machines is related to the capacity utilization of machines. Scheduling which is the last action in the production planning function, determines when and for how long a machine will be occupied with the production of specific parts or batches [8]. 
Dispatching deals with authorization of machine starts on various jobs based on the routing and scheduling. The action of controlling the start and stop of the works on the machines is called follow-up. Moreover, inspection of products acts as the problem detection method which will be fixed through the corrective actions on planning of the processes [7].

\section{$2.3 \quad$ Literature gap}

The current literature on the impact of DDM on PPC is quite limited and mostly is focused on the utilization of PPC practices also on direct digital manufacturing technologies [2]. However, there is no critical study of the PPC to evaluate the actual necessity of those concepts in the new production management [7]. We tend to address this literature gap through this paper.

\section{Method}

The methodology chosen for this research is the literature review and use of case studies while available. By doing so we aim to locate and describe the potential changes that are required in the current production planning and control practices as the DDM evolves. The current challenge with studying the DDM technologies is the lack of case data in the low or medium volume production which makes it difficult to study this phenomenon fully. However, by finding cases in somehow similar and currently in use production technologies we can expand our understanding of what might be possible when the existing limitations are removed. However, the prospects are bright as the new wider applications for the DDM is starting to appear which makes it possible for our method to be tested and refined against the facts while they become available.

\section{Results}

In this section we utilize case analysis and literature to synthesize a potential impact of DDM on the current PPC practices. Cases will be explained and then analyzed for the potential efficiency improvements and lessons learned in production planning and control of the future.

Our first case is an example of a digitalized manufacturing system. The company which is active in the area of custom-made industrial hoses has developed a production system which can be assumed as a digital factory. When the company receives the orders, they directly go to a machine which holds different variants of hoses with various diameters. The machine reads the order and chooses the correct hose by reading the tag on it. Then the ordered length will be cut and the process continues until the whole order batch is ready. While the machine is cutting the hose it also tags it with the correct metal connection ordered for it, which after the completing of the cutting on the production batch will be 
installed by the operator. Throughout this process the production planning has a shorter routing, simpler scheduling and loading. Moreover in this plant the digitalization has also enabled the possibility of customization on product by product bases. The main advantage of this process also comes from this feature which eliminates the need for stock keeping units as the orders can be manufactured and stocked in single containers with the customers tags.

In this case the dispatching can also be out-sourced to the machine through the prioritization of each order before sending it to the machine. Then if the machine receives an order with a higher priority it can utilize its capacity to fulfill the newly received one and hold the previous batch until the completion of that. In our case the follow-up process can be done well in advance as the process time can be predicted right after the loading. However, at this point the inspection of the products should be done manually but in the future when the machine can also install the metal end connections to the hose; this can also be done by the digital system. All in all, the digitalization of manufacturing can significantly reduce the need for SKUs on the product bases and also improve order delivery and inventory levels.

In direct digital manufacturing where, the product is manufactured through a CAD file, most of the PPC information can be delivered to the machines and after the production is complete it can be directly delivered to the customer after packaging. The additional value of a direct digital manufacturing will be less complexity in the PPC operations while increases the possibility for customization.

In the second example which is the result of analyzing the Volvos Kalmar radical automobile assembly plant, as depicted in Figure 1. The company tried to revolutionize the production line and switch towards a more worker friendly and group oriented fixed position (the vehicles were carried on electric carriers on the plant floors in various directions reprogrammable by the assembly group). Moreover, by giving the workers a team and a single vehicle at each time, they also tried to make the workers skilled in multiple tasks and provide them with the feeling of responsibility for the product that they are completing. However, while the efficiency of production was improved as the workers got used to their tasks and become comparable with the production line concept, Volvo Company decided to close the Kalmar plant in 1994 [4, 5]. Among the main reason was the fact that group assembly in a single location required kitting of the assembly parts and transferring them to the location of production and to the working team. This was quite a time consuming and complex task which in relatively simpler in the production line concept $[5,6]$. However, the kitting process in the future can be done by the autonomous robots, while the production plan is sent to the additive manufacturing machines, located in the plant to produce them according to the daily production plan. Then the robots similar to Kiva system 
which are in use by Amazon company can directly deliver the parts on a just in time bases to the assembly teams for the installation.

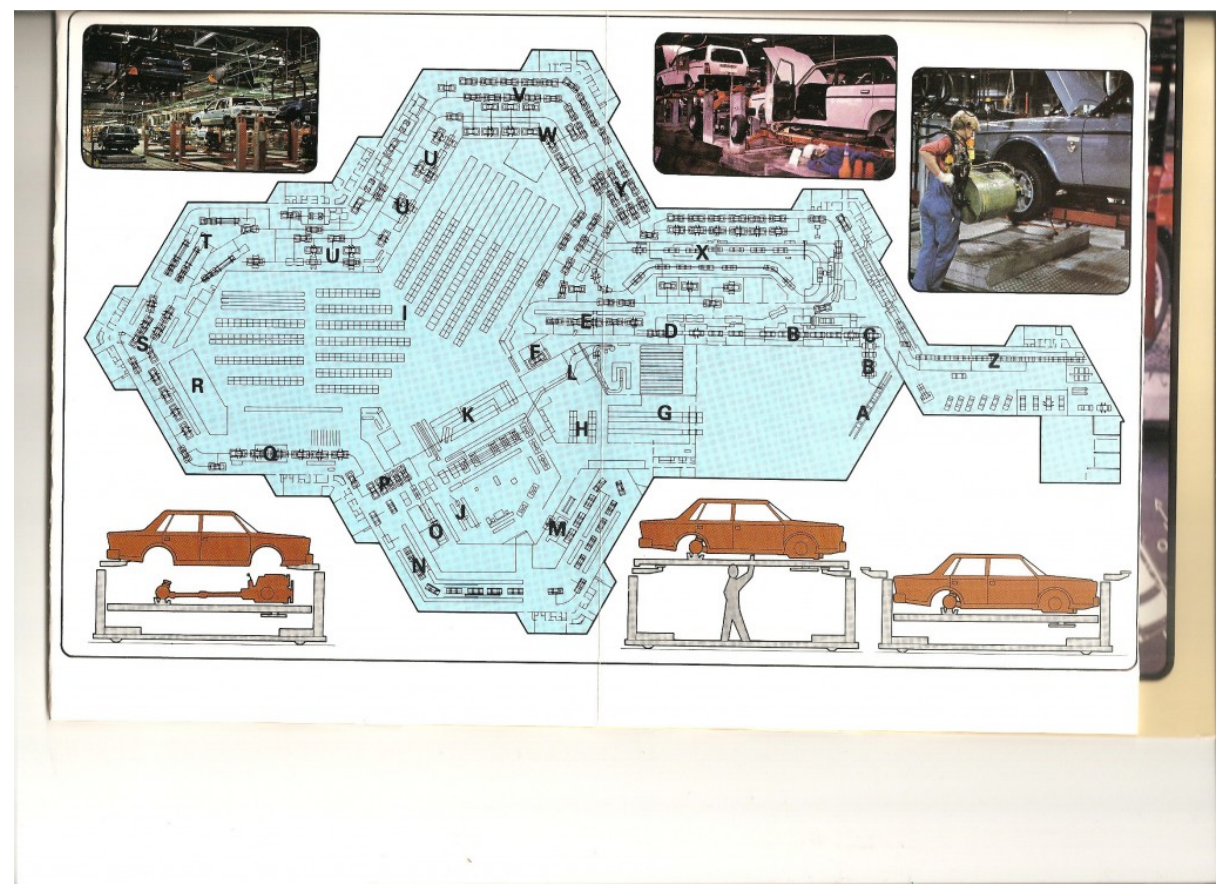

Fig. 1. Volvo Kalmar plant layout.

In such scenario, the complexity of kitting and dispatching will be out sourced to the computers and DDM systems and the follow-up as well as the product inspections can be done more carefully and on a timely fashion. Such scenario requires a major leap in the development of additive manufacturing technologies to enable a reliable and repeatable production method that can also produce the parts quicker and more economically. However, currently AM is suitable for a number of low volume productions such as jet engine components and medical prosthesis production but the push towards more customized products in various industries will make AM necessary for other ones. Also as the level of implementation grows, bigger industrial players consider the potential improvements to their business from direct digital manufacturing technologies and potentially to build a competitive edge, invest in the advancement and finding new applications. This potentially realizes the impact of DDM on production planning and control practices. 


\section{Conclusions}

Although the promise of DDM is strong and revolutionizing for the current manufacturing processes there is a need to understand the current limitations and possibilities. Although the DDM technologies are progressing rapidly, there still are sizable obstacles when it comes to the production throughput, reliability of parts, material range and costs. Having this in mind General Electric Company which is among the biggest and most advanced manufacturing companies in the world has started to manufacture the fuel nozzles of their CFM Leap jet engines using direct metal laser melting (DMLM) additive manufacturing process. And this is just the beginning as they and also other manufacturer of aerospace products are going to use this method for additional parts [3].

In this research we tried to utilize the industrial example to carefully predict the impact of DDM technologies on the PPC practices. That is important because production planning and control practices are the ones which are responsible for the efficiency of the plants as well as the on time delivery of the products with acceptable quality to the customer. Therefore, we think it is essential for these practices to become updated and be as efficient as possible.

The main limitation of this research is rooted in the lack of real world cases in the area of DDM which makes it hard to assess the situation fully. However, we see a significant opportunity for researchers to speculate and spread the possibilities of improvement to the industrial managers that they become aware of the potential for savings and improvements through this novel digital production technology.

\section{Referencies}

1. Khajavi, S. H., Partanen, J., Holmstrm, J. (2014). Additive manufacturing in the spare parts supply chain. Computers in Industry, 65(1), 50-63.

2. Gibson, I., Rosen, D. W., Stucker, B. (2010). Additive manufacturing technologies. New York: Springer.

3. Zaleski, A., (2015). GEs bestsellnig jet engine makes 3-D printing a core component. Fortune, Available at: http://fortune.com/2015/03/05/ge-engine3d-printing/

4. Sandberg, . (1995). Enriching production: Perspectives on Volvo's Uddevalla plant as an alternative to lean production.

5. Sandberg, T. (1995). Volvo Kalmartwice a pioneer. Enriching production, 87.

6. Engstrm, T., Jonsson, D., Johansson, B. (1996). Alternatives to line assembly: some Swedish examples. International Journal of Industrial Ergonomics, 17(3), 235-245.

7. Berry, W. L., Whybark, D. C., Jacobs, F. R. (2005). Manufacturing planning and control for supply chain management. New York: McGraw-Hill/Irwin. 
8. Peterson, R., Silver, E. A. (1979). Decision systems for inventory management and production planning (pp. 799-799). New York: Wiley.

9. Holmstrm, J., Romme, A. G. L. (2012). Guest editorial: Five steps towards exploring the future of operations management. Operations Management Research, 5(1-2), 37-42.

10. Kruth, J. P., Leu, M. C., Nakagawa, T. (1998). Progress in additive manufacturing and rapid prototyping. CIRP Annals-Manufacturing Technology, $47(2), 525-540$. 